\title{
Evaluation of the relative validity of a Web-based food frequency questionnaire used to assess Soy Isoflavones and nutrient intake in adolescents
}

\author{
Gina Segovia-Siapco* ${ }^{*}$, Keiji Oda and Joan Sabaté
}

\begin{abstract}
Background: Dietary assessment during adolescence is crucial in determining how adolescents' consumption habits potentially affect current and long-term health. However, assessment methods for adolescents need to be relevant to their emerging technological culture. We developed a web-based food frequency questionnaire (webFFQ) to assess the habitual soy isoflavones and nutrient intake of adolescents. Our purpose was to validate this webFFQ against multiple 1-day photograph-assisted food records (FR).

Methods: Adolescents aged 12-18 years $(n=70)$ attending middle and high schools completed the webFFQ and provided six 1-day FR. Fifteen participants were excluded due to improbable energy intake. Statistical agreements were determined using Wilcoxon signed-ranks test, Pearson's bivariate correlations of normalized values with energy-adjustment and correction for attenuation, cross-classification, and Bland-Altman plots.

Results: Based on $n=55$, the webFFQ had higher intake estimates for all isoflavones and most nutrients compared to the FR. Energy-adjusted and deattenuated correlations were moderately strong for total isoflavones $(r=0.67)$, daidzein $(r=0.63)$, genistein $(r=0.64)$, and glycitein $(r=0.54)$. They ranged from a high of 0.82 (animal proteins) to a low of 0.11 (vitamin B12) for nutrients. Cross-classification agreement up to within 1 quartile ranged from $99.0 \%$ (vegetable protein) to $61.8 \%$ (vitamin B12) with low gross misclassifications (0.0-12.7\%). Bland-Altman plots for the isoflavones showed consistent overestimation and wide variation but nevertheless good agreement between the two methods.
\end{abstract}

Conclusions: The webFFQ is relatively valid in ranking adolescents according to their isoflavones and nutrient intake. However, while it significantly overestimates the absolute intake of most nutrients, results are comparable to other food frequency questionnaires developed for adolescents.

Keywords: Adolescents, Dietary assessment, Dietary intake, Digital photographs, Evaluation, Food frequency questionnaire, Food records, Isoflavones, Soy, Validation, Web-based

\footnotetext{
* Correspondence: gsiapco@llu.edu

Center for Nutrition, Healthy Lifestyles and Disease Prevention, School of

Public Health, Loma Linda University, Loma Linda, CA 92350, USA
} 


\section{Background}

Most chronic diseases in adults originate from lifestyle practices and experiences during childhood and adolescence [1-3]. Meal-skipping [4-6] and snacking on highcalorie and low nutrient-dense foods are more common during adolescence, and these could be detrimental to their health [4-7]. Assessment of diet during this life stage is crucial in determining how consumption habits potentially affect current and long-term health. However, assessment of diet among the youth is laden with challenges. This group may have difficulty identifying or naming the foods they eat, conceptualizing food portion sizes, and recalling their intake due to limited food vocabulary; or, lack the patience, motivation, and perseverance to engage in dietary assessment activity $[8,9]$.

Despite their shortcomings, particularly recall bias and the need to be culture- or population-specific [10], food frequency questionnaires (FFQ) continue to be the mainstay in the dietary assessment of large study cohorts and in determining diet-and-health outcome relationships in adolescents [11]. FFQs are low-cost, time-efficient, easy to administer, can assess long-term or habitual intake, and entail less respondent burden compared to other dietary assessment methods.

A few validated FFQs for adolescents residing in the United States exist, mostly in the paper format [12-18]. Since a large percentage of adolescents spend a significant amount of time with technological devices, i.e., computers and mobile phones [19], the current technological revolution and emerging culture for young populations should be considered in the design of dietary assessment tools. Tools that tap into the technological skills of adolescents may keep them engaged and interested in dietary assessment activities [9]. In recent years, digital dietary assessment methods have been developed in an effort to improve the accuracy of food and nutrient consumption estimates [20] and decrease respondent burden in quantifying food intake [21]. Thus far, a few online FFQs have been developed for adolescents [22-25]. However, none of the existing FFQs, paper-based or online, met our need to assess the isoflavone intake of a multi-ethnic adolescent group, 25\% of whom are vegetarians and highly exposed to soy-containing foods.

We conducted the Teen Food and Development Study (TeenFADS) to investigate if soy isoflavones intake is associated with the health and development of adolescents. To make the dietary assessment more engaging and to improve compliance, we developed a web-based FFQ (webFFQ). Considering the context of ubiquitous use of mobile phones with a camera, the use of technology becomes more affordable in epidemiological studies if personal gadgets with unlimited texting and calling are employed. Thus, we chose multiple 1-day photographassisted food records (FR) as the reference comparison method and designed it such that participants used their own mobile phones to record their intake in text form and digital photographs. The purpose of this study was to validate the semi-quantitative webFFQ we developed for TeenFADS to estimate the habitual consumption of soy isoflavones and selected nutrients using multiple 1day FR as the reference method.

\section{Methods \\ Study design}

The TeenFADS is a cross-sectional study that investigated the associations between dietary intake and health and pubertal development of adolescents. Data were collected using an online questionnaire that consisted of several sections including a dietary assessment section (webFFQ) and school visits for the anthropometric measurements. This current study was designed to validate the webFFQ using multiple 1-day FR as reference method. Participants completed the webFFQ, underwent a one-on-one training to do FR, and then provided 61 day FR over 2-3 months. They utilized their personal mobile phones for their FR. The Institutional Review Boards of Loma Linda University (LLU) and Andrews University approved the study protocol and the informed consent process for both parental permission and assent of the adolescent participant.

\section{Study participants}

The TeenFADS involved adolescents aged 12-18 years who attended middle and high schools near Adventist universities in southern California and Michigan. Convenience sampling was done to recruit volunteers for the validation sub-study from among those who completed the webFFQ and attended the anthropometry clinic $(n=601)$. A question at the completion of the web-based questionnaire asked respondents if they were willing to participate in a sub-study. Those who responded positively were contacted and participants were selected based on the following criteria: (1) owned a mobile phone with good camera resolution; (2) had unlimited texting/calling; (3) after their one-on-one training, demonstrated through a return demonstration test the ability to follow video and/or printed instructions on how to take digital photographs correctly; and (4) could text messages and digital photographs to an email account set up for the study. Initially, 108 adolescents volunteered but of these, 23 did not meet the first two requirements. Of those who passed the training $(n=85)$, eight did not proceed with the study due to a busy schedule, five either lost or did not receive their fiducial markers and could not proceed with the return demonstration, and two lost interest in the study before it started. Thus, a total of 70 participants entered the study. 


\section{Web-based food frequency questionnaire}

The webFFQ is a 151-item self-administered, semiquantitative dietary assessment questionnaire that was designed to assess the soy intake and habitual diet of adolescents. The food list was initially composed of foods from a pilot questionnaire constructed at LLU that was administered among adolescents to determine their soy foods intake. Foods deemed commonly eaten based on interviews of multiethnic adolescents were added to this list. Registered Dietitians (RDs) who work with adolescents reviewed the list to ensure that the food names used were appropriate for this population. A comprehensive list composed of $151 \mathrm{food}$ items, 36 of which were soy-containing, were included in the final version (see Additional file 1: Table S1). Food items were grouped into eight categories that constituted eight screens in the webFFQ. The webFFQ was then pre-tested on 25 adolescents of the same demographic characteristics but were not participants in the study. This was done to determine clarity, interpretation, length of completion time, and if respondents would use the pop-up feature for additional information about an item if they needed further clarification. No conceptual difficulties in filling out the webFFQ were reported and the majority of participants did not find it necessary to seek additional information about the food items. Technical issues with use of specific browsers were resolved before administering the webFFQ.

Foods in the webFFQ are categorized as convenience foods (32 items), protein-rich foods (29 items), starches/cereals (17 items), vegetables/fruits (21 items), dairy products (10 items), beverages ( 24 items), snacks/sweets (11 items), and soups/legumes (7 items). These food groups are divided over eight screens (see Additional file 2: Figure S1). Food items are arranged in such a way that various types of a specific food are grouped (e.g., dairy milk: regular, low-fat or non-fat). Protein-rich foods such as meats also have their meat alternative counterparts (e.g., chicken, vegechicken) (see Additional file 3: Figure S2). More information about the food and examples pop up when the pointer is hovered over the food name (see Additional file 4: Figure S3). Fixed portion sizes are based on familiar measuring devices, e.g., cup, tablespoon, 12-fluid ounce can, and others.

On the webFFQ, respondents are asked to self-report the frequency of their intake during the past month. Frequency of intake is selected from a drop-down list of frequency categories which are: never/rarely, 1-3 times per month, once per week, 2-4 times per week, 5-6 times per week, once per day, 2-3 times per day, and 4 or more times per day (see Additional file 4: Figure S3). For seasonal foods, e.g., certain fruits, "when in season" is included as frequency of intake category. To ensure that no item is skipped, respondents are not allowed to move on to the next food category until all items on the screen are completely filled out. However, they can go back to make changes to their responses in any food category if desired.
The current variety of meat analog brands has various formulations. More specifically, some contain more gluten than soy protein or contain no soy protein at all. To differentiate between these formulations and more accurately determine soy and isoflavones intake, a separate window of different brand names for meat analogs appears for respondents to choose from when a frequency of "once per week" or greater is chosen. Frequency of "once per week" or greater for meat intake also shows a separate window where respondents choose the type of meat eaten (beef, chicken, turkey, lamb or pork). It takes approximately 25 min to complete the webFFQ.

\section{Food records with digital photographs}

Multiple day food records provide a better measure of food and/or nutrient intake and, thus, is commonly used as a criterion reference measure in evaluating or validating FFQs $[26,27]$. This dietary assessment method is often paperbased and recording is done on consecutive days. Weighing or taking a photograph of the foods before and after they are eaten are some of the approaches used to improve the accuracy of food records. As the criterion measure for this study, we determined the feasibility of using personal mobile phones for photographing foods to be consumed and recording intake as part of the food recording process among our young study participants. This method is described in a forthcoming article [28]. Instead of consecutive days of recording, the protocol was designed to reduce the burden associated with continuous food recording which may result in deviation from usual intake [27] or boredom, and to make the procedure more attainable among adolescents.

Before the data collection, participants were trained and then tested through a return demonstration of a one-day FR that was then evaluated to determine readiness, or additional training, for the study. During the trial runs, a few of the participants were excluded for losing their fiducial marker. Laminating the fiducial marker, adding a hole on one side and inserting a 2-foot long 1-inch wide ribbon through the hole resolved the problem of misplacing or losing the marker. Participants were randomly scheduled for six 1-day food recording days over a 2- to 3-month period. All five weekdays (Monday to Friday) and one weekend day (Sunday) were covered in scheduling the recording days.

The FR had two components: (1) digital photographs before- and after-intake of a meal that captures a fiducial marker for size approximation of objects in the digital photograph; and (2) text message that captures the time of intake, meal type (breakfast, snack, lunch, or dinner), and the foods eaten with corresponding amounts. Participants used their personal mobile phones to take digital photographs and record their intake. They sent their text report and photographs of their intake to a central location (i.e., the email address for the study). Reports from each participant (all digital photographs and texts sent 
during their reporting day) were reviewed and collated into food records the following day by trained research assistants. The amount of intake reported in the text was compared with the before- and after-eating digital photographs (after-eating digital photographs were only sent if there were leftovers) to determine if amount reported was estimated accurately or needed to be adjusted. If reports were incomplete, unclear, have discrepant information or other issues, the participant was contacted for clarification. Otherwise, the FR was cleared and logged on as completed.

\section{Isoflavones and nutrient intake determination}

Dietary intake reports on both the webFFQ and FR were coded using the Nutrition Data System for Research (NDSR) software version 2012 developed by the Nutrition Coordinating Center, University of Minnesota, Minneapolis, MN, for nutrient composition and analysis. The NDS$\mathrm{R}$ has a comprehensive database of about 18,000 foodswhich reflects variations according to preparation methods and component ingredients-and 163 nutrients/nutrient ratios/other food components derived from the USDA database and several other sources [29]. The comprehensive meat analogs database of the Adventist Health Study-2 [30] was utilized in determining the nutrient profile of meat analogs. Frequency values of average consumption during the past month were converted into frequency of intake per day, e.g., frequency of $1-3$ per month $=2 / 30=0.067$, and so on. Thus, factors used were 0 for "never/rarely", 0.067 for "1-3 times per month", 0.143 for "once per week", 0.429 for "2-4 times per week", 0.786 for "5-6 times per week", 1.0 for "once per day", 2.5 for "2-3 times per day", and 5.0 for " $\geq 4$ times per day". Nutrient amounts were relative to the fixed portion/serving sizes in the webFFQ. For vegetables that are eaten either raw or cooked, the average of raw and cooked nutrient components was used. For mixed foods, recipes were created by trained RDs and nutrient composition was based on the proportion of ingredients in the recipe. For single food items where several types exist, the NDS-R software computes nutrient composition for the most commonly eaten type (the "default" food), a determination made using nationally representative market research data [29]. Nutrient and isoflavones intake per day was computed afterwards using the product-sum method [27]. Daily FRs were coded on NDS-R separately for each participant.

\section{Statistical methods}

All 70 participants selected for this validation sub-study completed six recording days. However, 15 participants had to be excluded from the analyses: four due to improbable intake on the webFFQ which we predefined as $>4500 \mathrm{kcal}$ or $<1000 \mathrm{kcal}$ for boys and $>3500 \mathrm{kcal}$ or $<900 \mathrm{kcal}$ for girls, and 11 due to poor or non-compliance with the protocol in food recording. Thus, the analyzable data was based on information from 55 participants.

Most of the nutrients and isoflavones had skewed distributions. Absolute median intake estimates from the webFFQ and the FR were compared using Wilcoxon signed-ranks test. Intake values were either log- or square root-transformed to achieve normality before Pearson's bivariate correlation analyses were performed. Correction for within-person variation in multiple FR measurements was performed using the formula $r_{c}=r_{o}$ $\left[1+\left(\mathrm{S}_{\mathrm{w}}^{2} / \mathrm{nS}_{\mathrm{b}}^{2}\right)\right]^{1 / 2}$ [31] where $r_{c}=$ corrected correlation coefficient, $r_{o}=$ crude correlation coefficient between FFQ and mean of the multiple food records, $S_{\mathrm{w}}^{2}=$ within-person variance of the multiple food records, $S_{b}^{2}=$ estimate of the between person variance in the reference method (food records), and $n=$ number of repeated measures of the food records. Using this method for deattenuation or correction of the correlation coefficients creates conditions in which normally distributed errors for $r_{c}$ cannot be assumed. Thus, instead of the traditional asymptotic methods to determine confidence intervals about $r_{c}$, we computed 'distribution-free', non-parametric $95 \%$ confidence intervals using the bias-corrected and accelerated ( $\mathrm{BCa}$ ) bootstrap re-sampling method [32], with each confidence interval determined from the distribution of $r_{c}$ 's from 2000 samples.

Analytical tests were performed without energy adjustment and with energy adjustment using the residual method. The ability of the webFFQ to rank respondents according to intake was tested by determining classification agreement between quartiles of consumption levels according to webFFQ responses and the mean of the multiple FR. Proportions of exact agreement, within 1 quartile agreement, and gross misclassification were also estimated. Additional graphical determination of agreement with the reference method using Bland-Altman plots was done for the soy isoflavones (total, daidzein, genistein, and glycitein). Total isoflavones was computed as the sum of daidzein, genistein, and glycitein. Analyses were performed using the Statistical Analysis System (SAS) statistical software package version 9.4 (SAS Institute Inc., Cary, NC, USA) and R version 3.1.2 [33].

\section{Results}

Participants in the study included 33 girls and 22 boys with a mean age of 15.3 (standard deviation, $\mathrm{SD}=1.7$ ) years. Forty-six percent of the participants were Caucasians and the mean body mass index (BMI) z-score was $0.49(\mathrm{SD}=1.03)$. The analyzable group $(n=55)$ had similar characteristics as the entire study population except for the proportion of vegetarians (see Table 1). Those who were excluded did not significantly differ from the analyzable group (not shown). 
Table 1 Demographic characteristics of the study population

\begin{tabular}{llll}
\hline $\begin{array}{l}\text { Demographic } \\
\text { Characteristic }\end{array}$ & $\begin{array}{l}\text { Total Group } \\
(\text { TeenFADS) } \\
(N=601)\end{array}$ & $\begin{array}{l}\text { webFFQ Validation } \\
\text { Sub-Study } \\
\text { Group }(n=55)\end{array}$ & $p$-value \\
\hline $\begin{array}{l}\text { Age in years, mean (SD) } \\
\text { Gender }\end{array}$ & $15.0(1.8)$ & $15.3(1.7)$ & 0.17 \\
$\quad$ Males, \% & 44 & 40 & 0.57 \\
Females, \% & 56 & 60 & 0.57 \\
Ethnicity & & & \\
Caucasian, \% & 34 & 46 & \\
African/Afr-Am, \% & 9 & 6 & \\
Hispanic, \% & 14 & 16 & \\
Asians, \% & 11 & 7 & 0.66 \\
Others, \% & 7 & 7 & \\
Mixed, \% & 18 & 18 & 0.002 \\
Site & & & 0.24 \\
California, \% & 55 & 58 & \\
Michigan, \% & 45 & 42 & \\
Vegetarian, \% & 24 & 42 & \\
BMl z-scores, mean (SD) & $0.35(0.95)$ & $0.49(1.03)$ & \\
\hline
\end{tabular}

Table 2 shows the comparison of isoflavones and nutrient intake estimates between the webFFQ and the average of six 1-day FR using the Wilcoxon signed ranks test before and after energy adjustment. For most of the nutrients, estimates according to the webFFQ were higher compared to FR but not significantly for trans fats, carbohydrate, vitamin $\mathrm{E}$ and caffeine even after energy adjustment. WebFFQ estimates of isoflavones were about $300 \%$ more than the FR estimates.

Table 3 shows the correlation coefficients before and after adjustment for energy. Deattenuated correlations for energy-adjusted soy isoflavones ranged from 0.63 to 0.67 . In general, energy adjustment improved the correlation values except for folate, vitamin B12, calcium, iron, and zinc. Deattenuation after energy-adjustment further improved the correlations between the two methods for most of the nutrients except zinc, calcium, folate and iron-which remained the same or increased very slightlyand vitamin B12, which decreased to a non-significant value that was also the lowest correlation. Deattenuated energy-adjusted correlations between the two methods were weak (i.e., $r<0.35)$ for vitamin $\mathrm{B} 12(r=0.11)$ and zinc $(r=0.26)$; moderate (i.e., $r$ between 0.35 and 0.50$)$ for calcium, folate, vitamin $\mathrm{C}$, iron, vitamin $\mathrm{E}$, retinol, and protein; and strong $(r>0.50)$ for beta carotene, total fat, saturated fatty acids (SFA), monounsaturated fatty acids (MUFA), polyunsaturated fatty acids (PUFA), trans fats, animal protein, vegetable protein, and dietary fiber. Corrections for measurement error in the FR showed that within-person variances were higher than between-person variances, an indication that this group of adolescents have a wide variation in their day-to-day intake.

To further determine if the webFF provides a valid ranking of isoflavones and nutrient intake, agreement between quartile rankings on both methods was performed through cross-classification analysis (Table 4). Exact matches were moderate for soy isoflavones (38$49 \%$ ) and high for matches within 1 quartile (80-84 \%). For the nutrients, exact match was lowest for vitamin B12 (25.5 \%) and highest for animal protein (52.7 \%) while matches within one quartile ranged from $61.8 \%$ (vitamin B12) to $99.0 \%$ (vegetable protein). Gross misclassifications ranged from $0 \%$ (total isoflavones, animal and vegetable proteins, and vitamin E) to $12.7 \%$ (zinc). Results of the cross-classification analyses were consistent with the correlation analyses results.

Figure 1 shows the Bland-Altman graphs for total isoflavones, daidzein, genistein, and glycitein. The difference between estimates on the webFFQ and FR was plotted against the mean of the estimates of the two methods. All graphs show that the mean difference line is above zero, which indicates that the webFFQ consistently overestimated intake relative to FR. While a few of the dots appear closer to zero, more dots are scattered both above and below the mean difference between the two methods but very few of these dots are outside the limits of agreement (shaded space). The distribution of the dots also distinctly show non-consumers-shown as a few dots flocked at 0,0 -whereas the majority of the dots are spread out which indicates a larger proportion of this group were consumers with a wide variation in isoflavones intake (Fig. 1).

\section{Discussion}

The webFFQ is a comprehensive semi-quantitative questionnaire that was developed to assess isoflavones and nutrient intake of adolescents in a population presumably exposed to soy foods. We sought to evaluate the performance of this FFQ by comparing its absolute intake estimates, degree of agreement, and ranking ability with six days of 1-day FR as the reference method on soy isoflavones (total, daidzein, genistein, and glycitein), 20 nutrients, and caffeine. Overall, the webFFQ performed equivalently well as the FR in ranking the intake estimates for isoflavones and most of the nutrients. Corrected energy-adjusted correlations between the webFFQ and FR were moderately strong ( $r$ is between 0.50 and 0.70 ) for all the isoflavones and ranged from moderate $(r$ is between 0.3 and 0.5$)$ to strong $(r>0.5)$ for 18 of the 20 nutrients. Vitamin B12 and zinc were the only nutrients with weak correlations. Degree of agreement within one quartile was within the acceptable range (62\% for vitamin B12 to $99 \%$ for vegetable protein) and ranking ability was congruent for a substantial proportion $(>80 \%)$ of the participants 
Table 2 Comparison ${ }^{a}$ of reported soy isoflavones and nutrient intake on the web-based food frequency questionnaire with the reference method (food records with digital photographs) without energy adjustment and with energy adjustment $(n=55)$

\begin{tabular}{|c|c|c|c|c|c|c|c|c|c|c|}
\hline \multirow[b]{3}{*}{ Nutrient } & \multicolumn{5}{|c|}{ Without Energy Adjustment } & \multicolumn{5}{|c|}{ With Energy Adjustment } \\
\hline & \multicolumn{2}{|c|}{ Web-based FFQ } & \multicolumn{2}{|c|}{ Food Records } & \multirow[b]{2}{*}{$p$-value ${ }^{a}$} & \multicolumn{2}{|c|}{ Web-based FFQ } & \multicolumn{2}{|c|}{ Food Records } & \multirow[b]{2}{*}{$p$-value } \\
\hline & Median & $\mathrm{IQR}^{\mathrm{b}}$ & Median & IQR & & Median & IQR & Median & IQR & \\
\hline Energy, MJ & 8.77 & 6.29 & 7.63 & 2.56 & 0.02 & & & & & \\
\hline Total isoflavones, mg & 9.31 & 30.50 & 3.16 & 18.03 & $<0.001$ & 9.40 & 29.24 & 2.64 & 13.47 & $<0.001$ \\
\hline Daidzein, mg & 3.50 & 12.70 & 1.35 & 7.05 & $<0.001$ & 3.57 & 11.94 & 1.03 & 5.39 & $<0.001$ \\
\hline Genistein, mg & 4.98 & 15.37 & 1.68 & 9.37 & $<0.001$ & 4.92 & 14.82 & 1.36 & 7.09 & $<0.001$ \\
\hline Glycitein, mg & 0.82 & 2.96 & 0.27 & 1.50 & $<0.001$ & 1.07 & 2.70 & 0.27 & 1.24 & $<0.001$ \\
\hline Fat, g & 81.33 & 48.36 & 65.19 & 27.63 & $<0.001$ & 78.89 & 10.92 & 62.39 & 14.37 & $<0.001$ \\
\hline SFA, $g$ & 26.41 & 16.66 & 21.25 & 9.15 & $<0.001$ & 26.20 & 8.58 & 19.59 & 7.45 & $<0.001$ \\
\hline MUFA, g & 27.75 & 18.29 & 21.85 & 10.00 & 0.001 & 26.21 & 4.83 & 20.88 & 3.97 & $<0.001$ \\
\hline PUFA, $g$ & 21.50 & 12.93 & 17.05 & 7.06 & 0.001 & 20.01 & 7.28 & 15.79 & 4.65 & $<0.001$ \\
\hline Trans fats, $\mathrm{g}$ & 2.35 & 1.52 & 2.11 & 1.29 & 0.12 & 2.38 & 0.92 & 2.27 & 1.10 & 0.13 \\
\hline Carbohydrate, $g$ & 266.33 & 137.70 & 242.66 & 90.64 & 0.46 & 251.84 & 34.06 & 253.35 & 34.48 & 0.16 \\
\hline Protein, $\mathrm{g}$ & 80.66 & 57.78 & 62.40 & 28.15 & $<0.001$ & 76.46 & 11.29 & 62.99 & 16.59 & $<0.001$ \\
\hline Animal protein, $\mathrm{g}$ & 29.88 & 29.70 & 25.36 & 23.53 & 0.004 & 30.19 & 23.99 & 26.34 & 23.25 & $<0.001$ \\
\hline Vegetable protein, g & 44.88 & 27.37 & 35.92 & 21.10 & $<0.001$ & 44.80 & 19.45 & 35.63 & 12.66 & $<0.001$ \\
\hline Dietary fiber, g & 26.75 & 15.01 & 20.20 & 12.23 & $<0.001$ & 25.74 & 9.06 & 20.32 & 7.92 & $<0.001$ \\
\hline Beta carotene, mcg & 4038.70 & 3816.72 & 2547.64 & 2502.86 & 0.002 & 3629.93 & 2578.13 & 2432.11 & 2355.71 & 0.002 \\
\hline Retinol, mcg & 512.33 & 354.03 & 386.66 & 251.29 & $<0.001$ & 494.90 & 210.82 & 396.22 & 215.04 & $<0.001$ \\
\hline Vitamin E, mg & 10.07 & 6.57 & 9.42 & 4.57 & 0.56 & 9.03 & 3.09 & 9.69 & 3.33 & 0.06 \\
\hline Vitamin C, mg & 133.10 & 96.47 & 76.72 & 71.61 & $<0.001$ & 130.15 & 87.41 & 75.53 & 69.30 & $<0.001$ \\
\hline Folate, mcg & 606.31 & 374.12 & 422.31 & 208.30 & $<0.001$ & 578.85 & 195.57 & 439.31 & 165.14 & $<0.001$ \\
\hline Vitamin B12, mcg & 6.62 & 4.14 & 4.07 & 2.54 & $<0.001$ & 6.09 & 2.59 & 3.70 & 2.27 & $<0.001$ \\
\hline Calcium, mg & 1149.29 & 547.37 & 855.00 & 514.07 & $<0.001$ & 1125.58 & 400.07 & 839.31 & 382.86 & $<0.001$ \\
\hline Iron, mg & 17.82 & 8.30 & 14.21 & 6.32 & $<0.001$ & 17.23 & 3.80 & 14.07 & 3.38 & $<0.001$ \\
\hline Zinc, mg & 11.72 & 6.41 & 7.81 & 4.97 & $<0.001$ & 11.60 & 2.36 & 8.37 & 3.36 & $<0.001$ \\
\hline Caffeine, mg & 9.06 & 24.06 & 4.64 & 15.67 & 0.07 & 8.39 & 19.36 & 4.62 & 15.53 & 0.05 \\
\hline
\end{tabular}

Wilcoxon Signed Ranks test

${ }^{\mathrm{b}} \mathrm{QQR}$, interquartile range

SFA saturated fatty acid, MUFA monounsaturated fatty acid, PUFA polyunsaturated fatty acid

specifically for SFA, total protein, animal protein, vegetable protein, dietary fiber and the isoflavones (total isoflavones, daidzein, genistein, glycitein). However, compared to the FR, the webFFQ had significantly higher estimates of absolute intakes of energy, isoflavones and all nutrients, except carbohydrates, vitamin E, trans fats and caffeine.

In view of the findings regarding the use of technology in the dietary assessment of young people [9], we found a high completion rate of the webFFQ (only $2 \%$ of the participants did not complete). This could be attributed to the privacy, confidentiality of responses, and the convenience of filling out the questionnaire at home, at school, or where access to a computer and the internet was available.

Written food records and 24-h recalls are usually employed as standards in evaluating and validating FFQs. Given that digital photographs of foods eaten were obtained, using FR as the reference method allowed triangulation when evaluating portion size estimates and in identifying carelessly omitted food items in the texted food report. Considering that digital photographs improve energy and macronutrient intake estimates when combined with dietary food records [34], FR is certainly an appropriate alternative to the conventional written food record for this age group. Moreover, the FR afforded a cost-effective technology-based comparison method given that participants were willing to use their personal phone with a built-in camera and unlimited texting.

Relative to two other web-based [24, 25] and six paperbased [35-40] FFQs for adolescents which were validated against either multiple 24-h recalls or food records, our webFFQ shares similar overestimation bias for most nutrients. Of these eight FFQs, all have higher estimates for 
Table 3 Validity correlations between the webFFQ and FR estimates of nutrients and soy isoflavones, before and after energy adjustment ( $n=55$ adolescents)

\begin{tabular}{|c|c|c|c|c|c|c|c|c|}
\hline \multirow[t]{2}{*}{ Nutrient } & \multicolumn{3}{|c|}{ Without energy adjustment } & \multicolumn{5}{|c|}{ With energy adjustment } \\
\hline & Crude $r^{\dagger}$ & Corrected $r$ & $\mathrm{BCa} 95 \% \mathrm{Cl}^{\mathrm{a}}$ & Crude $r$ & $\mathrm{~S}_{\mathrm{w}}^{2 a}$ & $\mathrm{~S}_{\mathrm{b}}^{2 \mathrm{a}}$ & Corrected $r$ & BCa $95 \%$ C \\
\hline Energy, kcal & 0.32 & 0.37 & $(0.04,0.62)$ & & & & & \\
\hline Total isoflavones, mg & 0.58 & 0.65 & $(0.44,0.80)$ & 0.59 & 1.272 & 0.753 & 0.67 & $(0.44,0.81)$ \\
\hline Daidzein, mg & 0.54 & 0.61 & $(0.36,0.77)$ & 0.55 & 0.733 & 0.413 & 0.63 & $(0.38,0.79)$ \\
\hline Genistein, mg & 0.56 & 0.63 & $(0.40,0.80)$ & 0.56 & 0.881 & 0.491 & 0.64 & $(0.42,0.79)$ \\
\hline Glycitein, mg & 0.46 & 0.53 & $(0.26,0.73)$ & 0.48 & 0.193 & 0.099 & 0.56 & $(0.27,0.74)$ \\
\hline Fat, $g$ & 0.33 & 0.39 & $(0.12,0.66)$ & 0.59 & 0.060 & 0.026 & 0.69 & $(0.44,0.88)$ \\
\hline SFA, $g$ & 0.38 & 0.43 & $(0.12,0.69)$ & 0.66 & 0.124 & 0.076 & 0.74 & $(0.52,0.88)$ \\
\hline MUFA, $g$ & 0.32 & 0.40 & $(0.14,0.68)$ & 0.55 & 0.096 & 0.027 & 0.69 & $(0.34,0.92)$ \\
\hline PUFA, $g$ & 0.39 & 0.50 & $(0.18,0.79)$ & 0.43 & 0.176 & 0.032 & 0.59 & $(0.22,0.81)$ \\
\hline Trans fats, $\mathrm{g}$ & 0.28 & 0.37 & $(-0.02,0.80)$ & 0.54 & 0.693 & 0.195 & 0.68 & $(0.32,0.89)$ \\
\hline Carbohydrates, g & 0.36 & 0.40 & $(0.11,0.63)$ & 0.56 & 0.023 & 0.010 & 0.66 & $(0.37,0.84)$ \\
\hline Protein, $\mathrm{g}$ & 0.37 & 0.43 & $(0.18,0.62)$ & 0.39 & 0.053 & 0.027 & 0.45 & $(0.19,0.64)$ \\
\hline Animal protein, g & 0.65 & 0.70 & $(0.50,0.85)$ & 0.77 & 0.452 & 0.550 & 0.82 & $(0.67,0.91)$ \\
\hline Veg protein, g & 0.59 & 0.65 & $(0.50,0.77)$ & 0.72 & 0.080 & 0.069 & 0.79 & $(0.64,0.89)$ \\
\hline Dietary fiber, $g$ & 0.49 & 0.53 & $(0.35,0.65)$ & 0.65 & 0.103 & 0.092 & 0.71 & $(0.49,0.85)$ \\
\hline Beta carotene, mcg & 0.30 & 0.37 & $(0.05,0.62)$ & 0.44 & 2.127 & 0.484 & 0.57 & $(0.24,0.76)$ \\
\hline Retinol, mcg & 0.25 & 0.28 & $(-0.14,0.64)$ & 0.36 & 0.937 & 0.529 & 0.41 & $(0.08,0.72)$ \\
\hline Vitamin E, mg & 0.15 & 0.18 & $(-0.45,0.65)$ & 0.34 & 0.226 & 0.086 & 0.41 & $(0.01,0.77)$ \\
\hline Vitamin C, mg & 0.22 & 0.24 & $(-0.05,0.52)$ & 0.35 & 0.768 & 0.367 & 0.40 & $(0.08,0.65)$ \\
\hline Folate, mcg & 0.36 & 0.40 & $(0.17,0.59)$ & 0.32 & 0.139 & 0.070 & 0.38 & $(0.11,0.57)$ \\
\hline Vitamin B12, mcg & 0.26 & 0.30 & $(0.05,0.55)$ & 0.09 & 0.246 & 0.098 & 0.11 & $(-0.26,0.40)$ \\
\hline Calcium, mg & 0.35 & 0.39 & $(0.12,0.59)$ & 0.32 & 0.168 & 0.072 & 0.38 & $(0.09,0.63)$ \\
\hline Iron, mg & 0.40 & 0.47 & $(0.23,0.66)$ & 0.33 & 0.108 & 0.034 & 0.41 & $(0.18,0.60)$ \\
\hline Zinc, mg & 0.27 & 0.31 & $(-0.01,0.55)$ & 0.24 & 0.098 & 0.052 & 0.28 & $(0.01,0.49)$ \\
\hline Caffeine, mg & 0.39 & 0.49 & $(0.08,0.80)$ & 0.44 & 7.505 & 2.346 & 0.54 & $(0.10,0.83)$ \\
\hline
\end{tabular}

webFFQ web-based food frequency questionnaire, FR food records with digital photographs, SFA saturated fatty acid, MUFA monounsaturated fatty acid, PUFA polyunsaturated fatty acid

${ }_{r} r=$ Pearson's correlation coefficient where corrected $r$ is the value after correcting for multiple measurements in the reference methods; $\mathrm{S}_{\mathrm{w}}^{2}=$ within person variance in the multiple measures on the reference method; $S_{b}^{2}=$ between-person variance in the multiple measures on the reference method; $B C a$ (bias-corrected and accelerated) $95 \%$ confidence interval (CI) provides nonparametric confidence limits for the $r$. If interval does not include 0 , the correlation is statistically significant at alpha $=0.05$

dietary fiber, 7 overestimated energy and fat and 6 overestimated calcium. Out of seven FFQs that validated carbohydrate and protein, 6 overestimated these nutrients while 3 have higher estimates for iron. Three of the 4 FFQs that validated vitamin $C$ and retinol have higher estimates for these nutrients; and, out of three FFQs, two overestimated magnesium and saturated fat while one overestimated folate and monounsaturated fat.

Under-reporting of true intake in dietary food records [41, 42] coupled with overestimation tendencies during completion of lengthy FFQs may explain the lack of accuracy in the absolute intake estimates in this current study. Overestimation bias may also be attributed to inherent errors associated with the methods or the nature of the study population itself. Bias associated with under- reporting - by either a deliberate or careless omission of foods, or misreporting portions eaten, e.g., to avoid taking additional digital photographs for second helpings-and over-reporting in the webFFQ may explain the discrepancies between the two methods. Further investigation is needed to determine the sources of these biases.

One strength of this study was the use of a comparison method considered least likely to have similar errors inherent in FFQs, specifically reliance on memory [27]. Another strength was that the methods used were ageappropriate, less burdensome and less time-consuming for the respondent compared to the traditional paperbased food recording. In addition, digital photographs that accompanied the food reports allowed for adjustment of miscalculated portion sizes and identification of 
Table 4 Cross-classification of the ranked quartile nutrient and isoflavones intake estimates between the webFFQ and FR ( $n=55$ )

\begin{tabular}{|c|c|c|c|}
\hline Nutrient & $\%$ Exact match & $\%$ Exact match \pm 1 quartile & $\%$ Gross mismatch \\
\hline Energy, kcal & 32.7 & 69.1 & 7.3 \\
\hline Total isoflavones, mg & 45.5 & 81.8 & 0.0 \\
\hline Daidzein, mg & 49.1 & 83.6 & 1.8 \\
\hline Genistein, mg & 47.3 & 81.8 & 1.8 \\
\hline Glycitein, mg & 38.2 & 80.0 & 5.5 \\
\hline Fat, g & 29.1 & 79.0 & 1.8 \\
\hline SFA, $g$ & 41.8 & 80.0 & 1.8 \\
\hline MUFA, g & 27.3 & 76.4 & 9.1 \\
\hline PUFA, $g$ & 39.0 & 78.2 & 7.3 \\
\hline Trans fats, $g$ & 40.0 & 76.4 & 3.6 \\
\hline Carbohydrate, g & 38.2 & 70.9 & 3.6 \\
\hline Protein, $g$ & 32.7 & 80.0 & 3.6 \\
\hline Animal protein, g & 52.7 & 89.1 & 0.0 \\
\hline Vegetable protein, g & 47.3 & 99.0 & 0.0 \\
\hline Dietary fiber, $g$ & 49.1 & 83.6 & 1.8 \\
\hline Beta carotene, mcg & 34.5 & 69.1 & 9.1 \\
\hline Retinol, mcg & 29.1 & 65.5 & 7.3 \\
\hline Vitamin E, mg & 43.6 & 72.7 & 0.0 \\
\hline Vitamin C, mg & 40.0 & 72.7 & 3.6 \\
\hline Folate, mcg & 29.1 & 67.3 & 1.8 \\
\hline Vitamin B12, mcg & 25.5 & 61.8 & 10.9 \\
\hline Calcium, mg & 32.7 & 69.1 & 7.3 \\
\hline Iron, mg & 34.5 & 79.0 & 3.6 \\
\hline Zinc, mg & 34.5 & 76.4 & 12.7 \\
\hline Caffeine, mg & 36.4 & 72.7 & 7.3 \\
\hline
\end{tabular}

webFFQ web-based food frequency questionnaire, FR food records with digital photographs, SFA saturated fatty acid, MUFA monounsaturated fatty acid, PUFA polyunsaturated fatty acid

missed foods, considering that two-dimensional images could be more accurate at estimating food volume and in identifying cooking methods than 3-dimensional images [43]. Another strength was the human intervention in collating the final dietary food record. The follow-up calls from our research nutritionists within the next $48 \mathrm{~h}$ after the reporting day further ascertained the accuracy of the reports and the details needed for food components not apparently seen in the pictures.

Our study has a number of limitations. In our attempt to reduce respondent burden, our intermittent collection of 1-day food records may have not captured the inherent daily variation in intake compared to that of continuous food recording [27]. However, our results showed higher within-person than between-person variances in the 61 day food records. This indicates that intermittent food records can still catch daily variation in intake. Another limitation, common to most validity studies, is the use of convenience sampling and a small sample size. For this reason, we collected six FRs per participant to improve the precision of the comparison method in capturing habitual intake. Another limitation is the lack of a biochemical marker to assess isoflavones intake. However, it was already demonstrated that FFQ isoflavones estimates are significantly correlated with urinary isoflavonoids in a cohort of adults that includes parents of some of these adolescents [30]. The use of digital photographs in food records made this method less burdensome to respondents; however, managing the texts/digital photographs and quality control of the digital photograph-assisted FR on the part of the research team was still time-consuming [28]. Likewise, although human intervention in ascertaining the accuracy of food records submitted by participants is a strength, it is still subject to several errors, i.e., judgment, inter-evaluators, and coding. On the other hand, these limitations would not differ from managing food records collected through conventional methods.

Recall of dietary intake is more challenging for adolescents than adults so using the most recent past and shorter time coverage may reduce recall bias in 


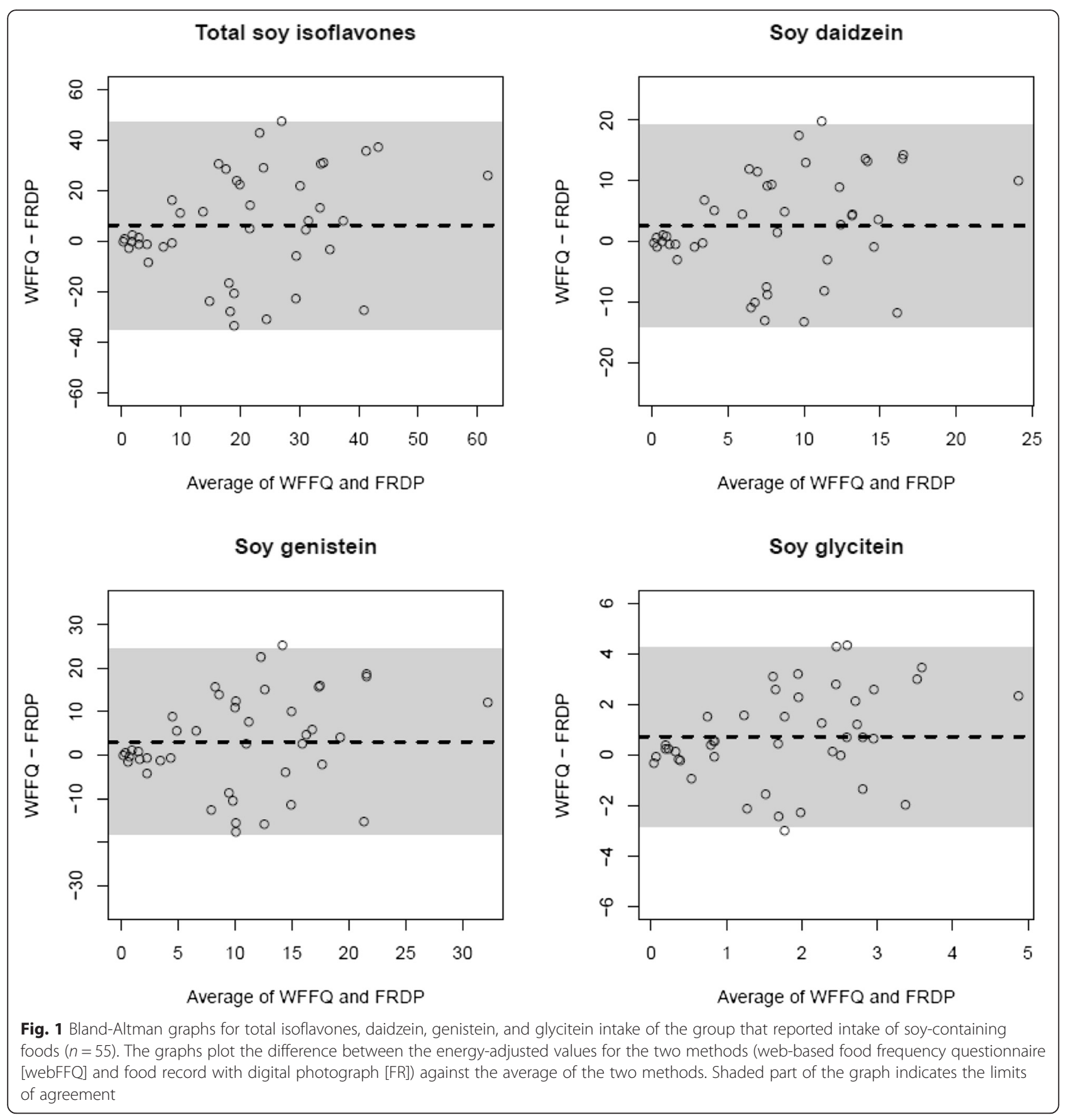

adolescents. This also reduces the burden of quantifying and averaging intake based on fixed portion sizes. However, this approach has its adjoining disadvantages, including the reduced ability to capture seasonal variations in the diet. We were not able to do a repeat administration of our webFFQ to determine if reported intakes are reproducible. A reproducibility study could have established confidence in the ability of our webFFQ to measure usual intake especially when the FFQ was designed to measure intake of the most recent past.

\section{Conclusions}

Compared to multiple 1-day food record with digital photographs, the 151-item webFFQ we developed for the TeenFADS is relatively valid in ranking adolescents according to their intake of soy isoflavones and nutrients. However, this web-based tool significantly overestimates adolescents' absolute intakes of soy isoflavones and most of the selected nutrients. Overall, the webFFQ may be used to classify adolescents in this population according to their dietary intake. 


\section{Additional files}

Additional file 1: Table S1. List of foods included in the web-based food frequency questionnaire (webFFQ). (DOCX $19 \mathrm{~kb}$ )

Additional file 2: Figure S1. Screen shot of the web-based food frequency questionnaire. (PDF $117 \mathrm{~kb}$ )

Additional file 3: Figure S2. Snapshots of the Beverage and Protein Foods screens. The respondent uses the pull-down menu to indicate frequency of intake of the specified food portion size. Foods are classified according to types available, e.g., regular or diet soda for beverages and real meat or meat alternative counterpart for protein foods. (PDF 224 kb)

Additional file 4: Figure S3. Additional food information shows up when pointer hovers over the name of the food. (PDF $62 \mathrm{~kb}$ )

\section{Abbreviations}

$\mathrm{BCa}$, bias-corrected and accelerated; $\mathrm{BMI}$, body mass index; $\mathrm{Cl}$, confidence interval; FR, photograph-assisted food records; MUFA, monounsaturated fatty acid; NDS-R, Nutrition Data Systems for Research; PUFA, polyunsaturated fatty acid SD, standard deviation; SFA, saturated fatty acid; TeenFADS, Teen Food and Development Study; webFFQ, web-based food frequency questionnaire

\section{Acknowledgements}

The authors would like to acknowledge each Teen Food and Development Study participant and the team of research assistants and dietitians who helped with the data collection. We also thank Dr. Michelle Wien for her editorial assistance.

\section{Funding}

This study was partially supported by grants from the Soy Nutrition Institute and White Wave Foods, which took no part in any aspect of the study from conception to the preparation of the manuscript, and McLean Funds, Nutrition Department, Loma Linda University.

\section{Availability of data and materials}

Given that we still have several reports to write and submit for publication from this study, we will not be able to share our data.

\section{Authors' contributions}

JS conceived, designed and directed the study, and critically reviewed the manuscript; KO analyzed the data; GSS designed the study with JS, supervised the data collection and management, and drafted the manuscript. All authors contributed to the editing and approval of the submitted manuscript.

\section{Competing interests}

The authors declare that they have no competing interests.

\section{Consent for publication}

Not applicable.

\section{Ethics approval and consent to participate}

The study protocol and the informed consent process, which required parental permission and assent of the adolescents to participate in the study, were approved by the Institutional Review Boards of Loma Linda University and Andrews University.

Received: 12 February 2016 Accepted: 15 June 2016

Published online: 08 July 2016

\section{References}

1. World Health Organization, Food and Agriculture Organization. Diet nutrition, and the prevention of chronic diseases. Geneva: WHO; 2003. Report No.: WHO technical report series 916. Available from: http://apps. who.int/iris/bitstream/10665/42665/1/WHO_TRS_916.pdf.

2. Adair LS. Long-term consequences of nutrition and growth in early childhood and possible preventive interventions. Nestle Nutr Inst Workshop Ser. 2014;78:111-20.

3. Lynch J, Smith GD. A life course approach to chronic disease epidemiology. Annu Rev Public Health. 2005;26:1-35.
4. Mathias KC, Jacquier E, Eldridge AL. Missing lunch is associated with lower intakes of micronutrients from foods and beverages among children and adolescents in the United States. J Acad Nutr Diet. 2016;116(4):667-76.e6.

5. Pearson N, Williams L, Crawford D, Ball K. Maternal and best friends' influences on meal-skipping behaviours. Br J Nutr. 2012;108(5):932-8.

6. Custers $K$, Van den Bulck J. Television viewing, computer game play and book reading during meals are predictors of meal skipping in a cross-sectional sample of 12-, 14- and 16-year-olds. Public Health Nutr. 2010;13(4):537-43.

7. Savige G, Macfarlane A, Ball K, Worsley A, Crawford D. Snacking behaviours of adolescents and their association with skipping meals. Int J Behav Nutr Phys Act. 2007;4:36.

8. Livingstone MBE, Robson PJ, Wallace JMW. Issues in dietary intake assessment of children and adolescents. Br J Nutr. 2004;92 Suppl 2:S213-22.

9. Boushey CJ, Kerr DA, Wright J, Lutes KD, Ebert DS, Delp EJ. Use of technology in children's dietary assessment. Eur J Clin Nutr. 2009:63 Suppl 1:S50-7.

10. Satija A, Yu E, Willett WC, Hu FB. Understanding nutritional epidemiology and its role in policy. Adv Nutr. 2015;6(1):5-18.

11. Rockett HR, Colditz GA. Assessing diets of children and adolescents. Am J Clin Nutr. 1997;65(4 Suppl):1116S-22.

12. Blum RE, Wei EK, Rockett HR, Langeliers JD, Leppert J, Gardner JD, et al. Validation of a food frequency questionnaire in Native American and Caucasian children 1 to 5 years of age. Matern Child Health J. 1999;3(3):167-72.

13. Buzzard IM, Stanton CA, Fiqueiredo M, Fries EA, Nicholson R, Hogan CJ, et al. Development and reproducibility of a brief food frequency questionnaire for assessing the fat, fiber, and fruit and vegetable intakes of rural adolescents. J Am Diet Assoc. 2001;101(12):1438-46.

14. Cullen KW, Watson K, Zakeri I. Relative reliability and validity of the Block Kids Questionnaire among youth aged 10 to 17 years. J Am Diet Assoc. 2008;108(5):862-6.

15. Di Noia J, Contento IR. Use of a brief food frequency questionnaire for estimating daily number of servings of fruits and vegetables in a minority adolescent population. J Am Diet Assoc. 2009:109(10):1785-9.

16. Harnack LJ, Lytle LA, Story M, Galuska DA, Schmitz K, Jacobs Jr DR, et al. Reliability and validity of a brief questionnaire to assess calcium intake of middle-school-aged children. J Am Diet Assoc. 2006;106(11):1790-5.

17. Hoelscher DM, Day RS, Kelder SH, Ward JL. Reproducibility and validity of the secondary level School-Based Nutrition Monitoring student questionnaire. J Am Diet Assoc. 2003:103(2):186-94.

18. Perks SM, Roemmich JN, Sandow-Pajewski M, Clark PA, Thomas E, Weltman A, et al. Alterations in growth and body composition during puberty. IV. Energy intake estimated by the youth-adolescent food-frequency questionnaire: validation by the doubly labeled water method. Am J Clin Nutr. 2000;72(6):1455-60.

19. Anderson JQ, Rainie L. The Future of the Internet. Washington, D. C. Pew Internet and American Life Project, Elon University School of Communications; 2012.

20. Stumbo PJ. New technology in dietary assessment: a review of digital methods in improving food record accuracy. Proc Nutr Soc. 2013;72(1):70-6.

21. Chiquete E, Ruiz-Sandoval JL, Ochoa-Guzman A, Sanchez-Orozco LV, LaraZaragoza EB, Basaldua N, et al. The Quetelet index revisited in children and adults. Endocrinol Nutr. 2014;61(2):87-92.

22. Hanning RM, Royall D, Toews JE, Blashill L, Wegener J, Driezen P. Webbased Food Behaviour Questionnaire: validation with grades six to eight students. Can J Diet Pract Res. 2009;70(4):172-8.

23. Matthys C, Pynaert I, De Keyzer W, De Henauw S. Validity and reproducibility of an adolescent web-based food frequency questionnaire. J Am Diet Assoc. 2007;107(4):605-10.

24. Overby NC, Johannesen E, Jensen G, Skjaevesland AK, Haugen M. Test-retest reliability and validity of a web-based food-frequency questionnaire for adolescents aged 13-14 to be used in the Norwegian Mother and Child Cohort Study (MoBa). Food Nutr Res. 2014:58.

25. Vereecken CA, De Bourdeaudhuij I, Maes L. The HELENA online food frequency questionnaire: reproducibility and comparison with four 24-h recalls in Belgian-Flemish adolescents. Eur J Clin Nutr. 2010;64(5):541-8.

26. Cade J, Thompson R, Burley V, Warm D. Development, validation and utilisation of food-frequency questionnaires - a review. Public Health Nutr. 2002;5(4):567-87.

27. Willett W. Nutritional Epidemiology. 3rd ed. New York: Oxford University Press; 2013

28. Segovia-Siapco G, Sabaté J. Feasibility of using personal mobile phones to assess dietary intake in adolescents. JMIR Mhealth Uhealth (forthcoming). doi:10.2196/mhealth.5418. 
29. Nutrition Coordinating Center University of Minnesota. Nutrition Data System for Research (NDS-R) 2013. Minneapolis: The Nutrition Coordinating Center, Division of Epidemiology, School of Public Health, University of Minnesota; 2000. p. 2003.

30. Jaceldo-Siegl K, Fraser GE, Chan J, Franke A, Sabate J. Validation of soy protein estimates from a food-frequency questionnaire with repeated $24-\mathrm{h}$ recalls and isoflavonoid excretion in overnight urine in a Western population with a wide range of soy intakes. Am J Clin Nutr. 2008;87(5): 1422-7.

31. Beaton GH, Milner J, Corey P, McGuire V, Cousins M, Stewart E, et al. Sources of variance in 24-hour dietary recall data: implications for nutrition study design and interpretation. Am J Clin Nutr. 1979;32(12):2546-59.

32. Efron B, Tibshirani RJ. An Introduction to the Bootstrap. New York: Chapman and Hall; 1993.

33. Team R. A language and environment for statistical computing. Vienna: R Foundation for Statistical Computing; 2015.

34. Ptomey LT, Willis EA, Goetz JR, Lee J, Sullivan DK, Donnelly JE. Digital photography improves estimates of dietary intake in adolescents with intellectual and developmental disabilities. Disabil Health J. 2015;8(1):146-50.

35. Ambrosini GL, de Klerk NH, O'Sullivan TA, Beilin LJ, Oddy WH. The reliability of a food frequency questionnaire for use among adolescents. Eur J Clin Nutr. 2009:63(10):1251-9.

36. Araujo MC, Yokoo EM, Pereira RA. Validation and calibration of a semiquantitative food frequency questionnaire designed for adolescents. J Am Diet Assoc. 2010;110(8):1170-7.

37. Rockett HR, Breitenbach M, Frazier AL, Witschi J, Wolf AM, Field AE, et al. Validation of a youth/adolescent food frequency questionnaire. Prev Med. 1997;26(6):808-16.

38. Slater B, Philippi ST, Fisberg RM, Latorre MRDO. Validation of a semiquantitative adolescent food frequency questionnaire applied at a public school in Sao Paulo Brazil. Eur J Clin Nutr. 2003;57(5):629-35.

39. Watanabe M, Yamaoka K, Yokotsuka M, Adachi M, Tango T. Validity and reproducibility of the FFQ (FFQW82) for dietary assessment in female adolescents. Public Health Nutr. 2011;14(2):297-305.

40. Watson JF, Collins CE, Sibbritt DW, Dibley MJ, Garg ML. Reproducibility and comparative validity of a food frequency questionnaire for Australian children and adolescents. Int J Behav Nutr Phys Act. 2009;6:62.

41. Burrows TL, Martin RJ, Collins CE. A systematic review of the validity of dietary assessment methods in children when compared with the method of doubly labeled water. J Am Diet Assoc. 2010;110(10):1501-10.

42. Gemming L, Rush E, Maddison R, Doherty A, Gant N, Utter J, et al. Wearable cameras can reduce dietary under-reporting: Doubly labeled water validation of a camera-assisted $24 \mathrm{~h}$ recall. Br J Nutr. 2014;1-8.

43. Chung LM, Chung JW. Tele-dietetics with food images as dietary intake record in nutrition assessment. Telemed J E Health. 2010;16(6):691-8.

\section{Submit your next manuscript to BioMed Central and we will help you at every step:}

- We accept pre-submission inquiries

- Our selector tool helps you to find the most relevant journal

- We provide round the clock customer support

- Convenient online submission

- Thorough peer review

- Inclusion in PubMed and all major indexing services

- Maximum visibility for your research

Submit your manuscript at www.biomedcentral.com/submit
) Biomed Central 